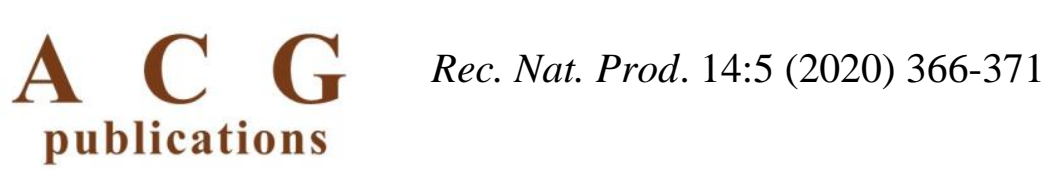

records of natural products

\title{
Essential Oil Composition of Tilia platyphyllos Scop. Collected from Different Regions of Kosovo
}

\author{
Nita Kelmendi $\odot^{1}$, Behxhet Mustafa $\odot^{2}$, Fitore Zahiri $\odot^{2}$ \\ Dashnor Nebija $\bigodot^{3 *}$ and Avni Hajdari $\odot^{2}$ \\ ${ }^{1}$ Department of Pharmacy, College of Medical Sciences "Rezonanca” Glloku te Shelgjet 10000- \\ Prishtina, Kosovo \\ ${ }^{2}$ Department of Biology. Faculty of Mathematical and Natural Science. University of Prishtina \\ "Hasan Prishtina". Mother Theresa St. 10000 Prishtina, Kosovo \\ ${ }^{3}$ Department of Pharmacy. Faculty of Medicine. University of Prishtina. "Hasan Prishtina"Mother \\ Theresa St. 10000 Prishtina, Kosovo
}

(Received February 13, 2020; Revised March 02, 2020; Accepted March 06, 2020)

\begin{abstract}
The aim of this study was to assess variation of the chemical composition of essential oils obtained from inflorescences and leaves of Tilia platyphyllos Scop. collected from five different localities in Kosovo. GCMS/GC-FID analysis revealed the presence of 96 compounds in essential oils obtained from inflorescences of large-leaved lime with the most prominent classes including hydrocarbons, with the respective concentrations in flowers and leaves of $41.7 \%-46.2 \%$ and $27.5 \%-36.8 \%$, respectively, oxygenated sesquiterpenes $(12.2 \%-23.0 \%$ and $21.3 \%-31.2 \%$, in flowers and leaves, respectively) and fatty acids and their derivatives $(8.0 \%-19.6 \%$ and $18.1 \%-23.1 \%$ in flowers and leaves, respectively) whereas monoterpenes were present in smaller amount. Experimental data showed that large-leaved lime is reach in different groups of volatile compounds, and this species could be further recommended for food and medicinal application.
\end{abstract}

Keywords: Large-leaved lime; essential oil; principal component analysis, (C) 2020 ACG Publications. All rights reserved.

\section{Plant Source}

Plant material (inflorescences) of the large-leaved lime (Tilia platyphyllos, Scop. Malvaceae), were collected from five different localities in Kosovo (Pejë, Gjilan, Lipjan, Klinë and Suharekë regions) during the period June-July 2016. The plant was identified by Professor asoc. Avni Hajdari, Department of Biology, University of Prishtina. A voucher specimen (Herbarium Number: LEB/2016/1) has been deposited at the Department of Biology University of Prishtina, Kosovo.

\section{Previous Studies}

Tilia platyphyllos Scop., Malvaceae, known as large-leaved lime or linden, is large-sized deciduous tree, native through Europe. This large-leaved tree prefers warmer climates and can reach $30-40 \mathrm{~m}$ in height, with a trunk up to around $1 \mathrm{~m}$ in diameter [1]. Asymmetrical, heart shaped leaves are

*Corresponding author: E-Mail: dashnor.nebija@uni-pr.edu Phone: + 383-45-401585 Fax: +383(0)38-512-223 
about $9 \mathrm{~cm}$ long and have pointed tips with upper surface of dark green colour and paler underside. The cream to yellow flowers, are fragrant, have five petals and hang in tiny clusters of 4 to 10 [2]. Largeleaved lime has industrial cosmetics and medicinal importance $[3,4]$. In Kosovo large leaved lime was traditionally used for different purposes, including tea preparation (recreational use/ panacea), treatment of sore throat and lung inflammations, abdominal pain and respiratory problems (cough, fever) [5-11]. It has been shown that the principal bioactive compounds of Tilia platyphyllos Scop. are mucilaginous substances, flavonoids and essential oils. [4, 11-13]. In a comparative metabolomics study of Tilia platyphyllos Scop. bracts totally 504 compounds were detected, including flavonoid glycosides, catechins, procyanidins, quinic acid derivatives and coumarins [14] . Chemical composition of essential oils obtained from linden inflorescences was studied in samples obtained from different region [15-19].

GC-FID and GC-MS analyses: GC analyses were performed using of an Agilent 7890A gas chromatography system equipped with flame ionization (FID) detector (Agilent Technologies) using HP-5MS column $30 \mathrm{~m} \times 0.25 \mathrm{~mm}$ with $0.25 \mu \mathrm{m}$ film thickness. Helium was used as the carrier gas with an initial flow rate of $0.6 \mathrm{~mL} / \mathrm{min}$ and subsequently at a constant pressure of $16.6 \mathrm{psi}$. GC/MS analyses were carried out using an Agilent 7800A GC System coupled with a 575C MSD. The ionisation energy was $70 \mathrm{eV}$ with a mass range of $40-400 \mathrm{~m} / \mathrm{z}$. The separation was performed with the same column and temperature program the same as for the analytical GC. Identification of the essential oils components was completed by comparing their Kovats retention indices with those reported in literature [20]. The components were also identified by comparing the mass spectra of each constituent with those stored in the MS library search (NIST 08.L and WILEY MS 9th) and with mass spectra from the literature [20].

Principal component analysis (PCA) was used to evaluate whether the identified essential oil components can be useful for reflecting the chemotaxonomy of Tilia platyphyllos. Constituents with concentrations higher than 3\% (bold in Table 1) of were subjected to statistical analysis.

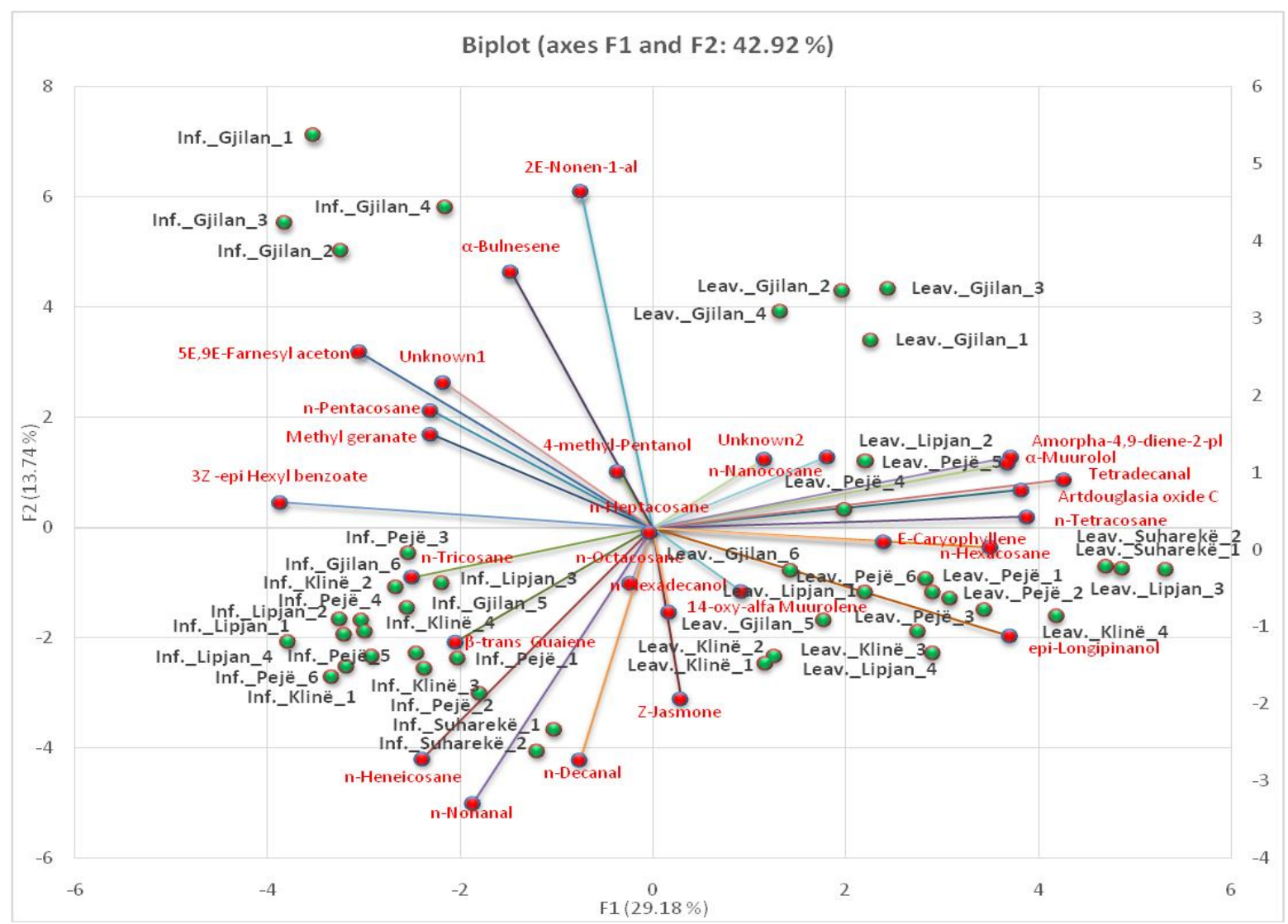

Figure 1. Graph obtained from the Principal Component Analysis of essential oils obtained from inflorescences of Tilia platyphyllos from 5 different localities. Inf. - Inflorescences; Leav. - leaves 
Table 1. The chemical composition of the essential oils obtained from the inflorescences (IF) and leaves (L) of Tilia platyphyllos

\begin{tabular}{|c|c|c|c|c|c|c|c|c|c|c|c|}
\hline \multirow[b]{2}{*}{$\mathbf{K} \mathbf{I}^{\mathbf{a}}$} & \multirow[b]{2}{*}{ Compound $^{b}$} & \multicolumn{2}{|c|}{ Pejë } & \multicolumn{2}{|c|}{ Gjilan } & \multicolumn{2}{|c|}{ Lipjan } & \multicolumn{2}{|c|}{ Klinë } & \multicolumn{2}{|c|}{ Suharekë } \\
\hline & & IF & $\mathbf{L}$ & IF & $\mathbf{L}$ & IF & $\mathbf{L}$ & IF & $\mathbf{L}$ & IF & $\mathbf{L}$ \\
\hline 838 & 4-methylpentanol & - & - & 1.42 & 1.00 & 1.63 & 2.50 & 1.02 & 0.65 & - & 0.20 \\
\hline 846 & $E$-2-Hexenal & 1.15 & 0.64 & 0.83 & 0.47 & 0.98 & 0.92 & 0.74 & 0.75 & 0.58 & 0.37 \\
\hline 862 & $E$-2-Hexenol & - & 0.05 & 0.44 & 0.29 & - & 0.07 & 0.03 & 0.20 & 0.85 & 0.46 \\
\hline 900 & $n$-Nonane & 0.16 & 0.03 & 0.19 & 0.04 & 0.26 & 0.14 & 0.21 & 0.37 & 0.17 & 0.14 \\
\hline 902 & Heptanal & 1.07 & 0.28 & 0.80 & 0.28 & 0.72 & 0.51 & 1.47 & 0.73 & 0.84 & 0.42 \\
\hline 960 & Benzaldehyde & 0.19 & 0.20 & 0.06 & 0.24 & 0.13 & 0.21 & 0.14 & 0.30 & - & 0.25 \\
\hline 973 & Hexanoic acid & 0.38 & 0.24 & 0.40 & 0.21 & 0.49 & 0.26 & 0.39 & 0.36 & 0.71 & 0.26 \\
\hline 987 & $E-3$-Hexenoic acid & 0.18 & 0.65 & 0.25 & 0.96 & 0.32 & 1.75 & 0.41 & 1.01 & 0.38 & 1.41 \\
\hline 989 & 1-Decene & 0.41 & 0.52 & 0.54 & 0.64 & 0.51 & 1.43 & 0.66 & 1.51 & 0.72 & 2.36 \\
\hline 988 & 2-Pentylfuran & 0.62 & 0.39 & 0.50 & 0.25 & 0.51 & 0.29 & 0.43 & 0.57 & 0.48 & 0.58 \\
\hline 1000 & $n$-Decane & 0.16 & 0.16 & 0.11 & 0.15 & 0.24 & 0.22 & 0.21 & 0.39 & - & 0.20 \\
\hline 1009 & Hexyl acetate & 0.77 & 0.56 & 0.52 & 0.56 & 0.63 & 0.69 & 0.50 & 0.70 & 0.42 & 0.64 \\
\hline 1035 & E-3-Octen-2-one & - & - & 0.46 & 0.43 & 0.36 & 0.52 & 0.35 & 0.70 & 0.20 & 0.35 \\
\hline 1042 & Benzeneacetaldehyde & 1.80 & 0.25 & 1.23 & 0.37 & 1.22 & 0.58 & 1.68 & 0.80 & 1.28 & 0.56 \\
\hline 1068 & n-octanol & 0.65 & 0.81 & 0.91 & 1.26 & 0.68 & 1.41 & 0.69 & 1.21 & 0.49 & 1.00 \\
\hline 1097 & 2-Nonanol & 0.34 & 0.87 & 0.40 & 0.66 & 0.50 & 1.90 & 0.41 & 0.72 & - & 0.33 \\
\hline 1097 & Linalool & 1.09 & 1.35 & 0.50 & 0.84 & 1.40 & 0.96 & 1.11 & 1.71 & 0.95 & 2.54 \\
\hline 1100 & $n$-Nonanal & 8.97 & 4.10 & $\mathbf{3 . 3 0}$ & 2.76 & 8.27 & 6.15 & 8.35 & 6.96 & 5.40 & 2.78 \\
\hline 1161 & 2E-Nonen-1-ol & 0.62 & 0.23 & 4.19 & 2.22 & 0.21 & 0.36 & 0.68 & 0.36 & - & 0.38 \\
\hline 1166 & Coahuilensol & 0.32 & 0.15 & 0.30 & 0.21 & 0.18 & 0.27 & 0.11 & 0.24 & - & 0.29 \\
\hline 1169 & Borneol & 0.32 & 0.11 & 0.42 & 0.14 & 0.55 & 0.23 & 0.16 & 0.05 & 0.30 & 0.21 \\
\hline 1175 & cis-Pinocamphone & 0.22 & 0.05 & 0.11 & 0.02 & 0.16 & 0.09 & 0.07 & 0.12 & 0.19 & 0.24 \\
\hline 1192 & 2-Decanone & 0.68 & 0.26 & 0.71 & 0.31 & 0.52 & 0.24 & 0.40 & 0.41 & 0.42 & 0.33 \\
\hline 1191 & Methyl salicylate & 0.72 & 0.23 & 0.62 & 0.24 & 0.54 & 0.22 & 0.33 & 0.34 & 0.27 & 0.20 \\
\hline 1196 & Safranal & - & 0.28 & - & 0.48 & - & 0.45 & 0.08 & 0.44 & - & 0.74 \\
\hline 1201 & $n$-Decanal & 0.81 & 0.48 & 0.44 & 0.53 & 0.68 & 0.65 & 0.97 & 0.90 & 3.00 & - \\
\hline 1219 & $\begin{array}{l}\beta \text {-Cyclocitral } \\
\text { 3Z-Hexenyl 3- }\end{array}$ & 0.46 & 0.28 & 0.49 & 0.28 & 0.23 & 0.37 & 0.26 & 0.25 & - & 0.35 \\
\hline 1235 & methylbutanoate & 0.20 & 0.13 & 0.72 & 0.23 & 0.63 & 0.23 & 0.26 & 0.27 & - & 0.32 \\
\hline 1258 & Carvenone & 1.61 & 0.05 & 1.40 & 0.33 & 1.38 & 0.28 & 0.72 & 0.39 & 0.81 & 0.36 \\
\hline 1270 & Nonanoic acid & 0.52 & 1.09 & 0.52 & 0.39 & 0.54 & 0.45 & 0.46 & 0.61 & 0.61 & 0.78 \\
\hline 1305 & Undecanal & 0.66 & 0.38 & 0.73 & 0.89 & 0.78 & 0.94 & 0.50 & 0.57 & - & 0.25 \\
\hline 1311 & 2-Adamantanone & - & 0.17 & 0.37 & 0.06 & 0.07 & 0.13 & - & - & - & 0.22 \\
\hline 1324 & Methyl geranate & 2.48 & 1.21 & 2.38 & 1.70 & 1.71 & 1.10 & 1.55 & 1.50 & 1.58 & 0.89 \\
\hline 1329 & Z-Hasmigone & 0.27 & 0.84 & 0.15 & 1.08 & 0.22 & 1.36 & 0.42 & 0.91 & 0.32 & 1.19 \\
\hline 1349 & $\alpha$-Terpinyl acetate & - & 0.08 & 0.17 & 0.26 & 0.03 & 0.10 & 0.18 & 0.79 & 0.61 & 1.08 \\
\hline 1359 & Eugenol & 0.23 & 0.60 & 0.17 & 0.09 & 0.18 & - & 0.18 & 0.15 & - & 0.31 \\
\hline 1366 & $n$-decanoic acid & 0.24 & - & 0.19 & - & 0.16 & - & 0.14 & 0.11 & 0.42 & 0.26 \\
\hline 1380 & Z-3-Hexenyl hexanoate & 0.39 & 0.17 & 0.21 & 0.09 & 0.24 & 0.11 & 0.27 & 0.54 & 0.43 & 0.79 \\
\hline 1384 & $E$ - $\beta$-damascenone & - & 0.18 & 0.15 & 0.21 & - & 0.22 & 0.05 & 0.18 & - & 0.33 \\
\hline 1391 & E-Jasmone & 0.18 & 0.49 & 0.30 & 0.56 & 0.08 & 0.11 & 0.05 & 0.14 & - & 0.15 \\
\hline 1392 & Z-Jasmone & 3.06 & 1.60 & 0.65 & 0.94 & 2.29 & 1.81 & 2.11 & 1.07 & 0.77 & 1.81 \\
\hline 1402 & $\alpha$-Funebrene & 0.42 & 0.07 & 0.57 & 0.09 & 0.12 & 0.16 & 0.27 & 1.27 & - & 0.26 \\
\hline
\end{tabular}


Table 1 continued..

\begin{tabular}{|c|c|c|c|c|c|c|c|c|c|c|c|}
\hline 1403 & Methyl eugenol & 0.25 & 0.66 & 0.02 & 0.01 & 0.04 & 0.35 & 0.12 & 0.11 & - & 0.47 \\
\hline 1419 & E-Caryophyllene & 0.18 & 0.78 & 0.14 & 0.74 & - & 1.11 & 0.09 & 0.72 & 1.07 & 4.20 \\
\hline 1422 & $\beta$-Duprezianene & 0.51 & 0.34 & 1.08 & 0.35 & 0.43 & 0.35 & 0.42 & 0.54 & 0.42 & 0.25 \\
\hline 1430 & $E$ - $\alpha$-Ionone & 0.32 & 0.09 & 0.15 & 0.15 & 0.38 & 0.06 & 0.25 & 0.32 & - & 0.21 \\
\hline 1441 & Aromadendrene & 0.15 & 0.02 & 0.06 & 0.32 & 0.13 & 0.53 & - & 0.07 & - & - \\
\hline 1446 & Seychellene & - & 0.41 & - & 0.35 & - & 1.47 & 0.09 & 0.50 & - & 1.04 \\
\hline 1454 & $\alpha$-Humulene & - & 0.98 & 0.34 & 0.94 & 0.71 & 0.08 & 0.44 & 0.11 & 0.35 & 0.22 \\
\hline 1466 & 2E-Dodecenal & 0.26 & 0.15 & 0.08 & 0.03 & 0.13 & 0.10 & 0.27 & 0.38 & - & - \\
\hline 1476 & $\alpha$-Neocallitropsene & 1.11 & 0.66 & 0.34 & 0.51 & 0.63 & 0.20 & 0.71 & 0.32 & 0.88 & 0.91 \\
\hline 1488 & $E$ - $\beta$-Ionone & - & 0.15 & 0.07 & 0.35 & 0.04 & 0.24 & 0.03 & 0.10 & - & 0.49 \\
\hline 1493 & $\beta$-etispirene & 0.18 & 1.30 & - & 1.37 & - & 1.66 & 0.20 & 0.78 & 0.39 & 0.86 \\
\hline 1500 & Isodaucene & - & 0.25 & 0.19 & 0.23 & - & 0.32 & 0.05 & 0.20 & - & 0.42 \\
\hline 1502 & trans- $\beta$-Guaiene & 1.30 & 0.25 & 1.71 & 0.70 & 2.72 & 0.48 & 2.64 & 0.78 & - & 1.27 \\
\hline 1509 & $\alpha$-Bulnesene & - & - & 4.64 & 0.38 & - & - & - & - & - & - \\
\hline 1512 & $\delta$-Z-Macrocarpene & 0.31 & 0.33 & 0.35 & 0.24 & 0.54 & 0.56 & 0.39 & 0.51 & 0.33 & 0.27 \\
\hline 1514 & $\alpha$-Cadinene & - & 0.39 & - & 0.42 & - & 0.45 & 0.05 & 0.17 & - & 0.88 \\
\hline 1520 & 1-endo-Bourbonanol & - & 0.37 & 0.01 & 0.31 & - & 0.35 & 0.07 & 0.26 & 0.31 & 0.52 \\
\hline 1523 & Artedouglasia oxide C & 0.44 & 1.62 & 0.56 & 1.27 & 0.35 & 1.50 & 0.62 & 1.55 & 0.37 & 2.59 \\
\hline 1536 & Liguloxide & - & 1.49 & 0.07 & 1.00 & - & 1.82 & 0.11 & 0.54 & - & 0.90 \\
\hline 1545 & $\alpha$-Calacorene & 0.16 & 0.77 & 0.19 & 2.16 & - & 1.63 & 0.24 & 0.80 & 0.26 & 0.43 \\
\hline 1563 & epi-Longipinanole & 1.26 & 4.05 & 0.82 & 2.08 & 1.74 & 6.96 & 2.33 & 5.42 & 2.02 & 7.82 \\
\hline 1567 & $3 Z$-epi Hexyl benzoate & 2.49 & 0.70 & 2.84 & 0.55 & 3.59 & 0.48 & 1.15 & 0.81 & 2.28 & 0.46 \\
\hline 1580 & $n$-Hexyl benzoate & - & 0.16 & - & 0.08 & - & 0.28 & 0.07 & 0.37 & - & 0.14 \\
\hline 1587 & $\begin{array}{l}\text { 2E-Hexenyl benzoate } \\
\text { 5-oxy-Isobornyl }\end{array}$ & - & 0.35 & - & 0.31 & - & 0.09 & 0.34 & 0.50 & 0.32 & 0.69 \\
\hline 1603 & isobutanoate & - & 0.33 & - & 0.10 & - & 0.08 & 0.05 & 0.26 & 0.78 & 0.48 \\
\hline 1612 & Tetradecanal & 0.45 & 3.34 & 0.60 & 3.72 & 0.58 & 4.12 & 1.34 & 3.63 & 0.70 & 4.67 \\
\hline 1646 & $\alpha$-Muurolol & 0.18 & 2.58 & 0.12 & 1.87 & - & 2.15 & 0.52 & 1.14 & 0.23 & 0.92 \\
\hline 1654 & $\alpha$-Cadinol & - & 0.20 & 0.24 & 0.19 & 0.30 & 0.13 & 0.48 & 0.21 & 0.26 & - \\
\hline 1668 & 6Z-Coniferyl alcohol & 0.36 & 0.45 & 0.29 & 0.58 & 0.13 & 0.41 & 0.19 & 0.58 & 0.35 & 0.59 \\
\hline 1673 & 5 iso-Cedranol & - & 0.56 & 0.14 & 0.82 & - & 0.37 & - & - & - & - \\
\hline 1676 & Cadalene & - & 0.36 & - & 0.17 & - & 0.11 & 0.27 & 0.07 & - & 0.26 \\
\hline 1700 & $n$-Heptadecane & 0.25 & 0.53 & 0.09 & 0.49 & 0.13 & 0.90 & 0.60 & 1.12 & 0.71 & 2.36 \\
\hline 1700 & Amorpha-4,9-dien-2-ol & 0.76 & 2.76 & 0.66 & 2.95 & 0.88 & 3.94 & 0.95 & 2.23 & - & 3.49 \\
\hline 1713 & $\begin{array}{l}\text { Cedroxyde } \\
\text { E-Sesquilavandulyl }\end{array}$ & - & 0.39 & 0.02 & 0.65 & - & 0.56 & 0.05 & 0.22 & - & 0.40 \\
\hline 1740 & acetate & 0.08 & 0.39 & - & 0.19 & - & 0.21 & 0.04 & 0.10 & 0.15 & 0.27 \\
\hline 1768 & Cedryl acetate & - & 1.01 & 0.18 & 1.10 & 0.48 & 0.81 & 0.38 & 0.53 & - & 0.95 \\
\hline 1768 & 14-oxy- $\alpha$ Muurolene & 1.76 & 1.09 & 1.34 & 2.15 & 0.86 & 2.50 & 1.26 & 1.11 & 3.35 & 1.01 \\
\hline 1788 & $\begin{array}{l}\text { 8-Cedren-13-ol, acetate } \\
\text { Isopropyl }\end{array}$ & 0.87 & 0.44 & 1.12 & 1.58 & 0.65 & 0.72 & 1.61 & 0.84 & 0.47 & 0.22 \\
\hline 1829 & $\begin{array}{l}\text { tetradecanoate } \\
2,7(14), 10-\end{array}$ & 0.61 & 0.64 & 0.73 & 0.60 & 0.97 & 1.15 & 0.96 & 1.66 & 0.64 & 0.41 \\
\hline 1845 & isabolatriene-1-ol-4-one & 0.26 & 1.64 & 0.04 & 0.82 & - & 0.53 & 0.04 & 0.36 & 0.47 & 0.70 \\
\hline 1847 & Phenylethyloctanoate & 0.21 & 0.39 & 0.21 & 0.27 & 0.39 & 0.27 & 0.35 & 0.87 & 0.67 & 0.32 \\
\hline
\end{tabular}


Table 1 continued..

\begin{tabular}{|c|c|c|c|c|c|c|c|c|c|c|c|}
\hline 1875 & $n$-Hexadecanol & 1.32 & 2.18 & 1.37 & 0.81 & 0.49 & 0.62 & 0.90 & 1.70 & 1.11 & 0.66 \\
\hline 1900 & n-Nanodecane & 2.79 & 1.76 & 1.22 & 1.45 & 2.28 & 1.01 & 1.41 & 0.56 & 0.26 & 0.23 \\
\hline 1913 & $5 E, 9 E$-Farnesyl acetone & 2.42 & 1.47 & 3.79 & 1.07 & 3.45 & 0.55 & 2.46 & 0.74 & 11.98 & 0.56 \\
\hline 2100 & $n$-Heneicosane & 13.50 & 4.07 & 3.59 & 2.10 & 12.38 & 2.16 & 4.92 & 5.94 & 9.88 & 3.91 \\
\hline 2300 & $n$-Tricosane & 7.25 & 3.16 & 5.48 & 1.73 & 5.92 & 0.46 & 21.76 & 2.89 & 5.20 & 0.93 \\
\hline 2400 & $n$-Tetracosane & 0.85 & 1.94 & 1.10 & 2.37 & 1.23 & 2.75 & 1.16 & 2.83 & 2.84 & 5.16 \\
\hline 2500 & $n$-Pentacosane & 13.90 & 10.88 & 19.61 & 13.58 & 14.65 & 8.91 & 10.75 & 9.85 & 1.06 & 0.49 \\
\hline 2600 & $n$-Hexacosane & 0.42 & 3.71 & 1.65 & 2.64 & 0.41 & 3.56 & 0.74 & 3.62 & 2.17 & 6.84 \\
\hline 2700 & $n$-Heptacosane & 1.14 & 2.40 & 1.48 & 4.21 & 1.70 & 0.96 & 1.00 & 1.10 & 2.72 & 1.81 \\
\hline 2725 & Unidentified 1 & 5.87 & 5.67 & 5.49 & 4.96 & 5.88 & 2.70 & 3.48 & 3.63 & - & 4.83 \\
\hline 2745 & Unidentified 2 & 0.24 & 1.51 & 0.35 & 2.00 & 0.28 & 0.92 & 0.18 & 0.32 & 1.33 & 0.49 \\
\hline 2800 & $n$-Octacosane & 1.74 & 2.74 & 2.96 & 2.26 & 1.36 & 1.55 & 0.98 & 1.38 & 6.47 & 1.77 \\
\hline 2900 & $n$-Nanocosane & 1.49 & 4.91 & 3.66 & 4.96 & 2.80 & 3.43 & 1.54 & 3.37 & 13.98 & 3.69 \\
\hline \multicolumn{2}{|c|}{ Total (\%) } & 99.9 & 100 & 100.0 & 100 & 99.9 & 100 & 100 & 99.9 & 99.9 & 100 \\
\hline
\end{tabular}

In this study, ninety-six compounds were separated (Table 1) from the essential oils obtained from inflorescences of Tilia platyphyllos, from which ninety-four were identified, while two compounds were not identified. (Supporting information, Figures S1 and S2). The most abundant classes of compounds were hydrocarbons with the respective concentrations in inflorescences and leaves of: $41.68-46.18 \%$ and $27.48-36.81 \%$; followed by oxygenated sesquiterpenes (12.19 $23.05 \%$ and $21.29-31.19 \%$ in inflorescences and leaves, respectively), fatty acids and their derivatives (8.03 - 19.61\% and $18.05-23.1 \%$ in inflorescences and leaves, respectively); sesquiterpenes (3.44 - 9.42\% and $6.04-10.84 \%$ in inflorescences and leaves, respectively) and oxygenated monoterpenes $(4.23 \%-6.18 \%$ and $3.88-7.22 \%$ in inflorescences and leaves, respectively) (Table 1). The results of our study were in accordance with studies performed elsewhere $[16,17,19]$. In this work the total phenolic and flavonoid contents and antioxidant activity of methanol extract of Tilia platyphyllos was studied as well (Supporting information, Table 1 and 2).

The two-dimensional axis system of the PCA (Figure 1) shows the presence of two principal groups of compounds clustered based on the plant organs from which volatile compounds have been extracted, while the origin of plant material played a smaller role in the variability of the chemical composition of essential oils.

\section{Supporting Information}

Supporting Information accompanies this paper on http://www.acgpubs.org/journal/recordsof-natural-products

\section{ORCID}

Nita Kelmendi:0000-0002-0346-3356

Behxhet Mustafa:0000-0002-1052-5252

Fitore Zahiri:0000-0003-4867-1906

Avni Hajdari:0000-0001-5688-9679

Dashnor Nebija:0000-0002-8853-794X 


\section{References}

[1] E. Eaton, G. Caudullo and D. De Rigo (2016). Tilia cordata, Tilia platyphyllos and other limes in Europe: distribution, habitat, usage and threats. In: European Atlas of forest tree species. eds: J. S.M.Ayanz, D. de Rigo, G. Caudullo, T, H. Durrant, A. Mauri Publication Office of the European Union, Luxembourg. pp: 184-185.

[2] S. Jensen (2003) EUFORGEN. Technical Guidelines for genetic conservation and use for lime (Tilia spp.). International Plant Genetic Resources Institute, Rome, Italy. 6 pages. European Forest Genetic Resources Programme. Available from http://www.euforgen.org/ Last accessed: 17.11.2019

[3] University of Connecticut Plant Database. M. H. Brand, Department of Plant Science and Landscape Architecture, Storrs, CT 06269-4. Available form: http://hort.uconn.edu/plants. Last accessed:16.11.2019

[4] M. Blumenthal (1998). American Botanical Council, Bundesinstitut für Arzneimittel und Medizinprodukte, Therapeutic Guide to Herbal Medicines, The Complete German Commission E monographs. American Botanical Council

[5] R. L. Sõukand, C. L. Quave, A. Pieroni, M. Pardo-de-Santayana, J. Tardío, R. Kalle, Ł. Łuczaj, I. Svanberg, V. Kolosova, L. Aceituno-Mata, G. Menendez-Baceta, I. Kołodziejska-Degórska, E. Pirożnikow, R. Petkevičius, A. Hajdari and B. Mustafa. (2013) Plants used for making recreational tea in Europe: a review based on specific research sites, J. Ethnobiol. Ethnomed. 9(1), 58 (12 pages).

[6] B. Mustafa, A. Hajdari, A. Pieroni, B. Pulaj, X. Koro and C.L. Quave (2015). A cross-cultural comparison of folk plant uses among Albanians, Bosniaks, Gorani and Turks living in south Kosovo, $J$. Ethnobiol. Ethnomed. 12;11:39 (26 pages).

[7] A. Pieroni, R. Sõukand, C.L. Quave, A. Hajdari and B. Mustafa (2017). Traditional food uses of wild plants among the Gorani of South Kosovo, Appetite 108, 83-92.

[8] A. Hajdari, A. Pieroni, M. Jhaveri, B. Mustafa and C.L. Quave (2018). Ethnopharmacological practices of slavic speaking people in South Kosovo, Ethnobiol. Conserv. 7(6), (42 pages).

[9] B. Mustafa, A. Hajdari, F. Krasniqi, E. Hoxha, H. Ademi, C.L. Quave, and A. Pieroni (2012). Medical ethnobotany of the Albanian Alps in Kosovo, J. Ethnobiol. Ethnomed. 8, 6 (14 pages).

[10] B. Mustafa, A. Hajdari, Q. Pajaziti, B. Syla, C.L. Quave and A. Pieroni (2012). An ethnobotanical survey of the Gollak region, Kosovo. Genet. Resour. Crop Ev. 69,739-754.

[11] European Medicines Agency. Available from https://www.ema.europa.eu/en Last accessed: 17.11.2019

[12] G. Toker, M. Aslan, E. Yeşilada, M. Memişoğlu and S. Ito (2001). Comparative evaluation of the flavonoid content in officinal Tiliaeflos and Turkish lime species for quality assessment, J. Pharmaceut. Biomed. Anal. 26(1),111-1121.

[13] A. Karioti, L. Chiarabini, A. Alachkar, M. Fawaz Chehna, F.F. Vincieri and A. R. Bilia (2014). HPLCDAD and HPLC-ESI-MS analyses of Tiliae flos and its preparations, J. Pharmaceut. Biomed. Anal. 100, 205-214.

[14] Z. Szücsa, Z. Cziáky, A. Kiss-Szikszai, L. Sinka, G. Vasas and S. Gonda (2019).Comparative metabolomics of Tilia platyphyllos Scop. bracts during phenological development, Phytochemistry 167,112084.

[15] L. Ahmadi and M. Mirza (1999). Volatile constituents of Tiliapla typhyllos Scop. flowers, J. Essent. Oil Res.11(6),765-766.

[16] G. Toker, K. H. C. Baser, M. Kürkçüoglu and T. Özek (1999). The composition of essential oils from Tilia L. species growing in Turkey, J. Essent. Oil Res. 11(3), 369-374.

[17] A. Prączko and J. Góra (2000). The chemical composition of essential oil distillated from Linden blossoms, Żywność 4(25), 90-94.

[18] V. Rădulescu and E. Oprea (2008). Analysis of volatile compounds of officinal Tiliae flos by gas chromatography coupled with mass spectrometry, Farmacia 56(2), 129-138.

[19] R. Kowalski, T.Baj, K.Kalwa, G. Kowalska and M. Sujka (2017) Essential oil composition of Tilia cordata flowers, J. Essent. Oil Bear. Pl. 20(4), 1137-1142.

[20] R. Adams (2007). Identification of essential oil components by gas chromatography/mass spectrometry, Allured, Carol Stream, Ill, USA, 4th edition.

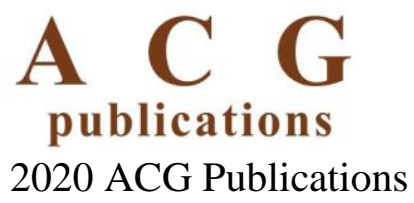

